\title{
First superburst from a classical low-mass X-ray binary transient
}

\author{
L. Keek ${ }^{1,2}$, J. J. M. in 't Zand ${ }^{1,2}$, E. Kuulkers ${ }^{3}$, A. Cumming ${ }^{4}$, E. F. Brown ${ }^{5}$, and M. Suzuki ${ }^{6}$ \\ 1 SRON Netherlands Institute for Space Research, Sorbonnelaan 2, 3584 CA Utrecht, The Netherlands \\ e-mail: 1.keek@sron.nl \\ 2 Astronomical Institute, Utrecht University, PO Box 80000, 3508 TA Utrecht, The Netherlands \\ ISOC, ESA/ESAC, Urb. Villafranca del Castillo, PO Box 50727, 28080 Madrid, Spain \\ 4 Physics Department, McGill University, 3600 rue University, Montreal, QC, H3A 2T8, Canada \\ 5 Department of Physics \& Astronomy, National Superconducting Cyclotron Laboratory, and the Joint Institute for Nuclear \\ Astrophysics, Michigan State University, East Lansing, MI 48824, USA \\ ${ }^{6}$ Institute of Space and Astronautical Science, JAXA, 1-1, Sengen 2chome, Tsukuba-city, Ibaraki 305-8505, Japan
}

Received 10 August 2007 / Accepted 19 November 2007

\begin{abstract}
We report the analysis of the first superburst from a transiently accreting neutron star system with the All-Sky Monitor (ASM) on the Rossi X-ray Timing Explorer. The superburst occurred 55 days after the onset of an accretion outburst in 4U 1608-522. During that time interval, the accretion rate was at least $7 \%$ of the Eddington limit. The peak flux of the superburst is 22 to $45 \%$ of the Eddington limit, and its radiation energy output is between $4 \times 10^{41}$ and $9 \times 10^{41} \mathrm{erg}$ for a distance of $3.2 \mathrm{kpc}$. Fits of cooling models to the superburst light curve indicate an ignition column depth between $1.5 \times 10^{12}$ and $4.1 \times 10^{12} \mathrm{~g} \mathrm{~cm}^{-2}$. Extrapolating the accretion history observed by the ASM, we derive that this column was accreted over a period of 26 to 72 . The superburst characteristics are consistent with those seen in other superbursting low-mass X-ray binaries. However, the transient nature of the hosting binary presents significant challenges for superburst theory, requiring additional ingredients for the models. The carbon that fuels the superburst is thought to be produced mostly during the accretion outbursts and destroyed in the frequent type-I X-ray bursts. Mixing and sedimentation of the elements in the neutron star envelope may significantly influence the balance between the creation and destruction of carbon. Furthermore, predictions for the temperature of the neutron star crust fail to reach the values required for the ignition of carbon at the inferred column depth.
\end{abstract}

Key words. X-rays: binaries - X-rays: bursts - X-rays: individuals: 4U 1608-522

\section{Introduction}

4U 1608-522 is a bright low-mass X-ray binary (LMXB) with a neutron-star (NS) primary (Belian et al. 1976; Tananbaum et al. 1976). QX Nor was identified by Grindlay \& Liller (1978) as the optical counterpart. The accretion onto the neutron star is transient (Lochner \& Roussel-Dupre 1994, and references therein) and the outburst behavior complex. Three main flux states can be resolved in 4U 1608-522 (Wachter et al. 2002): an outburst state, a low-intensity state and a true quiescent state. In contrast to many other LMXB transients, its true quiescent state has a relatively low duty cycle of only about $50 \%$. Wachter et al. speculate that the mass transfer rate might be fluctuating around the critical threshold separating stable accretion systems from true transient systems, possibly due to varying stellar spots on the donor star or to a varying vertical accretion disk structure which may change the irradiation and, thus, the mass transfer rate through the disk. Wachter et al. (2002) also identified a modulation in the optical light curve with a period of $12.9 \mathrm{~h}$ which they suspect to be near the orbital period. Thus, the donor is probably hydrogen-rich.

4U 1608-522 exhibits so-called type-I X-ray bursts (Belian et al. 1976; Tananbaum et al. 1976). These are understood to result from thermonuclear shell flashes on the surfaces of NSs of layers of accreted hydrogen and helium (Woosley \& Taam 1976; Maraschi \& Cavaliere 1977; for reviews, see Lewin et al. 1993, and Strohmayer \& Bildsten 2006). The flash layers heat up during a fraction of a second and cool down in an exponentiallike manner lasting $10 \mathrm{~s}$ to a few minutes. As a result, a spectrum may be observed which is typically well fit by a black body with a temperature decreasing from a peak of typically $k T=2$ to $3 \mathrm{keV}$ and an emission region similar in size to what is expected for a NS (Swank et al. 1977). Some nuclear energy generation may persist throughout the cooling phase, prolonging the bursts somewhat. A number of bursts exhibit photospheric radius expansion (PRE) where the burst flux is thought to reach the Eddington limit. From the observation of PRE bursts from 4U 1608-522, Galloway et al. (2006) derived a distance to this source of $3.2 \pm 0.3 \mathrm{kpc}$. During 5 PRE bursts, burst oscillations were detected at $619 \mathrm{~Hz}$, which implies the fastest known spin frequency for accreting neutron stars (Galloway et al. 2006), with the possible exception of XTE J1739-285 (Kaaret et al. 2007).

Since a few years a different kind of type-I X-ray burst is detected in about $10 \%$ of all bursters: so-called "superbursts" (Cornelisse et al. 2000; Strohmayer \& Brown 2002). These are about $10^{3}$ times more energetic and last for hours to a day. They are thought to result from flashes of carbon-rich layers (Strohmayer \& Brown 2002; Cumming \& Bildsten 2001). These layers are presumed to be much further down in the neutron star than those where ordinary X-ray bursts occur, which would explain the longer duration. It has been observed that superbursts influence the normal bursting behavior. Each time when the start of a superburst has been observed with sufficient statistics a precursor burst was seen (see e.g. Strohmayer \& Brown 2002). Furthermore, after the superburst the normal bursting behavior is 
quenched for approximately one month (see e.g. Kuulkers et al. 2002b).

Superbursts have so far only been seen in systems in which the NS has been continuously accreting for at least $10 \mathrm{yr}$ (Kuulkers 2004; in't Zand et al. 2004), although not in every such system (Keek et al. 2006). Here we present a superburst from 4U 1608-522, which represents the first case for a "classical" transient. A superburst has also been observed from the transient source KS 1731-260 (Kuulkers et al. 2002b). However, unlike KS 1731-260, 4U 1608-522 exhibits states of high flux with a duration that is short with respect to the expected superburst recurrence time. This may have interesting consequences for the theory of superbursts, because the different states of the transient allow us to study different levels of carbon production and burning in one source. Perhaps most importantly, whereas the crust in KS 1731-260 is expected to have been heated significantly out of thermal equilibrium with the core during its long outburst, the crust of $4 \mathrm{U} 1608-522$ is not expected to be significantly heated during its short outbursts. This presents a significant challenge to carbon ignition models for superbursts, which require a hot crust to achieve ignition at the depths inferred from observations of superbursts. Despite these differences, the superburst from $4 \mathrm{U} 1608-522$ has similar properties to superbursts from other sources.

The paper is organized as follows. First we describe the observations and spectral calibration in Sect. 2. In Sect. 3 we report on an analysis of the X-ray burst behavior of 4U 1608-522 as a function of its apparent mass accretion rate. Next, in Sect. 4, we present the analysis of the detected superburst. Finally, we discuss the implications of the results for superburst theory in Sect. 5. Initial reports of this superburst appeared in Remillard et al. (2005) and Kuulkers (2005).

\section{Observations and spectral calibration}

\subsection{Observations}

To study both the long-term accretion and bursting behavior of $4 \mathrm{U} 1608-522$, as well as the superburst, we employ observations performed with multiple instruments on-board four X-ray observatories.

The All-Sky Monitor (ASM) on the Rossi X-ray Timing Explorer (RXTE) (Levine et al. 1996) consists of three Scanning Shadow Cameras (SSC), each containing a position-sensitive proportional counter. The cameras are mounted on a rotating drive. Data are accumulated in so-called dwells of $90 \mathrm{~s}$. After each dwell the rotation drive changes the orientation of the SSCs. Spectral information is available from three channels with corresponding energy ranges of roughly $1.5-3,3-5$ and $5-12 \mathrm{keV}$. After the launch of RXTE in December 1996 until November 2006 , the ASM observed 4U 1608-522 for a total of 2.8 Ms. On May 5th 2005 the ASM observed the superburst from 4U 1608522.

RXTE also carries the Proportional Counter Array (PCA). The PCA consists of five proportional counter units (PCUs) with a total geometric area of $8000 \mathrm{~cm}^{2}$ and has a bandpass of 1 to $60 \mathrm{keV}$ (Jahoda et al. 2006). 4U 1608-522 was observed for $1.6 \mathrm{Ms}$ in total, mostly when it was out of quiescence (e.g., van Straaten et al. 2003; Gierliński \& Done 2002).

The BeppoSAX observatory (Boella et al. 1997a) was launched in April 1996 and carried two Wide Field Camera's (WFCs) as well as four Narrow Field Instruments (NFI). The WFCs (Jager et al. 1997) are coded mask aperture cameras with a band pass of 2-28 keV. During the six year lifespan of BeppoSAX a campaign of semi-yearly observations of the Galactic Center were carried out, which resulted in an exposure time of 3.8 Ms for $4 \mathrm{U}$ 1608-522.

The BeppoSAX NFI performed two pointed observations of $4 \mathrm{U}$ 1608-522. The first observation was performed during an outburst on February 28, 1998. In this paper we analyze the broad-band spectrum obtained with the following NFI in the indicated energy bands: the Low Energy Concentrator Spectrometer (LECS; Parmar et al. 1997; 0.12-4 keV), the Medium Energy Concentrator Spectrometer (MECS; Boella et al. 1997b; 1.8-10 keV) and the Phoswich Detection System (PDS; Frontera et al. 1997; 15-220 keV). The exposure time depends on the instrument and is $30 \mathrm{ks}$ for the MECS. The second observation took place when 4U 1608-522 was in quiescence. Since we are interested in the production of carbon from accreted matter, we forgo the analysis of the data from this observation.

On May 5th 2005, 33 min before the start of the ASM observation of the superburst, the High Energy Transient Explorer 2 (HETE-2; Ricker et al. 2003) observed a flare from 4U 1608522 with two instruments: the Wide field X-ray Monitor (WXM; Shirasaki et al. 2003) with a 2 to $25 \mathrm{keV}$ band pass and the French Gamma Telescope (FREGATE; Atteia et al. 2003) which has a band pass of 6 to $400 \mathrm{keV}$.

The International Gamma-Ray Astrophysics Laboratory (INTEGRAL; Winkler et al. 2003) was launched in October 2002. The Imager on Board the Integral Satellite (IBIS; Ubertini et al. 2003) is a coded aperture camera containing two detectors. We use results obtained with the INTEGRAL Soft Gamma-Ray Imager (ISGRI; Lebrun et al. 2003) detector, which has an energy range from 15 to about $500 \mathrm{keV}$. IBIS/ISGRI has observed $4 \mathrm{U} 1608-522$ up to September 2005 for a total of 6.3 Ms.

In this paper we analyze spectra using version 11.2 .0 of the XSPEC software package (Arnaud 1996).

\subsection{Spectral calibration of the ASM}

The spectral response of the ASM detectors is not well defined. A pre-flight effective area array is available, while a redistribution matrix is not (see discussion in Kuulkers 2002). We construct such a matrix by modeling, for an infalling photon with a certain energy, the energy distribution over the three channels by a Gaussian with a full width at half maximum of $20 \%$ of the centroid energy, which is a typical width for the type of proportional counter used in the ASM (e.g. Fraser 1989). The response matrix thus obtained is merely a first order estimate, but suffices for rough calculations.

We employ the Crab source to investigate the accuracy of our response matrix. The average count rate over all ASM observations from this source in the full $1.5-12 \mathrm{keV}$ band-pass is $75.4 \mathrm{c} \mathrm{s}^{-1}$. The X-ray spectrum can be described by an absorbed power law. Kirsch et al. (2005) performed simultaneous model fits to Crab spectra obtained with $22 \mathrm{X}$-ray instruments. Using the results of these fits in the $2-10 \mathrm{keV}$ range, our response matrix $^{1}$ predicts an ASM $1.5-12 \mathrm{keV}$ count rate of $70.5 \mathrm{c} \mathrm{s}^{-1}$. Therefore, when performing spectral analyses using this matrix, the normalization of the models needs to be corrected by a factor 1.07 to account for this discrepancy. Note that in principle this factor can be different for each of the three SSCs and can vary with time. However, we find that in a time interval of 100 days centered at the start of the superburst the difference in Crab count rate between any two SSCs never exceeds the $3 \sigma$ level.

\footnotetext{
1 Kuulkers (2002) finds the count rate is over-predicted. This is due to a different choice of values for the spectral model parameters.
} 


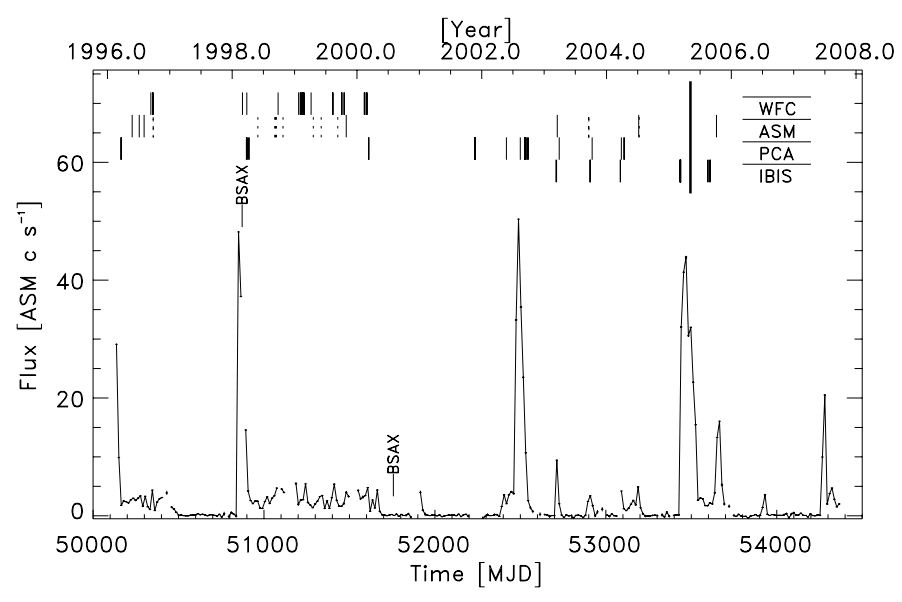

Fig. 1. 1.5-12 keV RXTE-ASM light curve of 4U 1608-522 at a 2-week time resolution. Data points with errors in excess of $0.5 \mathrm{c} \mathrm{s}^{-1}$ were excluded from this plot. The connecting line is broken if data points are more than 2 weeks apart. The vertical lines indicate 37 bursts detected with the WFC, 19 with the ASM (dashed lines indicate tentative bursts), 31 with the PCA (Galloway et al. 2006) and 19 with the IBIS/ISGRI (Chelovekov et al. 2007). The long vertical line indicates the time of the superburst. "BSAX" indicates the times of two BeppoSAX observations.

Apart from the accuracy in predicting the count rate of the $\mathrm{Crab}$, we also investigate how well our response matrix can reproduce the typical model parameters that are found by fitting an absorbed power law to the three-channel spectral data. We extracted a spectrum from all the ASM data on the Crab available at the time of writing. We fix the hydrogen absorption column density at the value of $N_{\mathrm{H}}=0.45 \times 10^{22} \mathrm{~cm}^{-2}$, as found by Kirsch et al. (2005) (in the $0.1-1000 \mathrm{keV}$ energy range). Following Kirsch et al., we use the abundances found by Wilms et al. (2000) and cross sections from Verner et al. (1996). Leaving free the power law index and the normalization, we do not find an acceptable agreement with the data. Only if we add in quadrature $10 \%$ of the flux to the uncertainty of each data point are we able to obtain an acceptable fit with $\chi_{\text {red }}^{2} \simeq 1$. Taking into account the correction factor derived previously, we find the best fit with a photon index of $\Gamma=2.01 \pm 0.12$ and a normalization of $N_{\text {powerlaw }}=9 \pm 2$ photons $\mathrm{keV}^{-1} \mathrm{~cm}^{-2} \mathrm{~s}^{-1}$, which is consistent with the results from Kirsch et al. (2005). The uncertainty in $N_{\text {powerlaw }}$ is large, because by definition $N_{\text {powerlaw }}$ is the photon flux at $1 \mathrm{keV}$, which is outside of the ASM energy range. The XSPEC power law model "pegpwrlw" uses a user-defined energy range for the normalization. Employing the ASM bandpass gives an uncertainty in the normalization of $6 \%$.

\section{History of accretion and X-ray burst activity}

\subsection{Long-term light curve}

Figure 1 shows the 1.5-12 keV ASM light curve of 4U 1608522. Clearly visible are the three states identified by Wachter et al. (2002; see Sect. 1). Four major outbursts, where the peak photon count rates was in excess of $20 \mathrm{c} \mathrm{s}^{-1}$ for more than one month, are visible in the 11-year time span of the observations (see Sect. 3.2 for a discussion on the selection criterion). The first outburst was ongoing at the start of the ASM observations and lasted until 60 days afterward, with fluxes in excess of $20 \mathrm{c} \mathrm{s}^{-1}$. The following outbursts lasted with fluxes above $20 \mathrm{c} \mathrm{s}^{-1}$ for
$44 \mathrm{~d}$ (MJD 50 848-50 892), $48 \mathrm{~d}$ (MJD 52 475-52523), and $80 \mathrm{~d}$ (MJD 53438-53518), respectively. There are also five minor outbursts, after the two latter major outbursts. The low-intensity states are visible in the first half of the mission. From comparing the first half and the latter half of the light curve, it appears that either a low-intensity state emerges after a major outburst, or a series of minor outbursts. This characteristic is only now apparent, after 11 of observation (Wachter et al. 2002 only considered the first 5 years of the data set). We determined the average flux over the complete ASM data to be $2.466 \pm 0.006 \mathrm{c} \mathrm{s}^{-1}$. This is about $2 \%$ of the flux reached during the brightest bursts seen in the ASM.

\subsection{Accretion rate}

When matter is accreted onto the neutron star surface, some of the accretion energy and/or some of the material may leave in a jet. If these losses are significant, the persistent flux is not a good tracer of the mass accretion rate $\dot{M}$. For black-hole X-ray binaries, Fender et al. (2005) argue that jets are not present during the high soft state. It is during the high soft state of 4U 1608522 that we are particularly interested in the precise value of the accretion rate. Therefore, assuming that the same holds true for neutron star binaries, in this paper we will assume there are no losses through a jet and that the persistent flux is a good measure for $\dot{M}$. Expressed as a fraction of the Eddington-limited mass accretion rate $\dot{M}_{\text {Edd }}$, which is $\dot{M}_{\text {Edd }}=2 \times 10^{-8} M_{\odot} \mathrm{yr}^{-1}$ for a canonical hydrogen-accreting neutron star with a mass of $1.4 M_{\odot}$, it can simply be derived as $\dot{M} / \dot{M}_{\text {Edd }}=F / F_{\text {Edd }}$, with $F$ the bolometric flux and $F_{\text {Edd }}$ the Eddington-limited flux. We will determine the bolometric flux below. The Eddington-limited flux is exhibited during photospheric radius expansion (PRE) bursts. RXTE PCA observed 12 such bursts (Galloway et al. 2006). The mean unabsorbed bolometric peak flux of these bursts is $F_{\text {Edd }}=(1.32 \pm 0.14) 10^{-7} \mathrm{erg} \mathrm{s}^{-1} \mathrm{~cm}^{-2}$.

Van Straaten et al. (2003) make an extensive study of the spectral changes of $4 \mathrm{U} 1608-522$ during the various states and the associated timing properties. They conclude that $4 \mathrm{U} 1608$ 522 is an Atoll source that exhibits the "banana" state during outbursts and moves in the color-color diagram to the "extreme island' state when the flux goes to levels of the low-intensity state. The distinguishing parameter is the "hard color" (i.e., the flux between 9.7 and $16.0 \mathrm{keV}$ divided by the flux between 6.0 and $9.7 \mathrm{keV}$ ) which is about 1.15 in the extreme island state and 0.55 in the banana state. This implies that one-band measurements, such as the ASM count rates, are not an accurate measure of the bolometric flux, let alone the accretion rate. In order to derive the bolometric flux during the low and high accretion states, we analyze the broad-band outburst-observation with BeppoSAX as well as a large number of observations with the RXTE PCA.

Two broad-band observations were carried out during the ASM coverage with BeppoSAX, one of which during outburst (for the timing of these observations, see Fig. 1). To determine the flux during this observation we analyze spectra obtained with the LECS, MECS and PDS. We subtract background spectra taken from blank field observations at the same detector positions for the LECS and MECS spectra, while for the PDS offsource pointings are used as background. The spectra are rebinned to obtain at least 15 photons in each bin while sampling the instrument resolution with at most 3 channels at all energies, to ensure the applicability of the $\chi^{2}$ statistic. An error of $1 \%$ is added in quadrature to the statistical error per bin to account for systematic uncertainties. This is common practice in BeppoSAX analyses (Fiore et al. 1999). We fit the data from all 
Table 1. Parameters and $1 \sigma$ uncertainties of fitting the outburst spectrum of $4 \mathrm{U}$ 1608-522 from BeppoSAX NFI observations on 28 Feb. 1998 with an absorbed disk black body ${ }^{a}$ and comptonized ${ }^{b}$ model. $^{2}$

\begin{tabular}{ll}
\hline \hline$N_{\mathrm{H}}$ & $(8.91 \pm 0.05) 10^{21} \mathrm{~cm}^{-2}$ \\
\hline$k T_{\mathrm{BB}}$ & $2.38 \pm 0.07 \mathrm{keV}$ \\
$N_{\mathrm{BB}}$ & $18.3 \pm 0.9$ \\
\hline$k T_{0}$ & $0.478 \pm 0.005 \mathrm{keV}$ \\
$k T_{\mathrm{e}}$ & $3.6 \pm 0.2 \mathrm{keV}$ \\
$\tau$ & $3.7 \pm 0.2$ \\
$N_{\text {Comptt }}$ & $0.40 \pm 0.02$ \\
& $1.40 / 160$ \\
$\chi_{\text {red }}^{2} /$ d.o.f. & $(1.75 \pm 0.10) \times 10^{-8} \mathrm{erg} \mathrm{s}^{-1} \mathrm{~cm}^{-2}$ \\
Flux $(0.01-300 \mathrm{keV})^{c}$ & $39.4 \pm 0.9 \mathrm{c} \mathrm{s}^{-1}$ \\
\hline ASM count rate & \\
\hline
\end{tabular}

${ }^{a}$ Disk black body model parameters: $k T_{\mathrm{BB}}$ is the temperature at the inner disk radius $R_{\text {in }}$ and $N_{\mathrm{BB}} \equiv\left(R_{\mathrm{in}}^{2} / d_{10}^{2}\right) \cos \theta$ the normalization, with $R_{\text {in }}$ in units of $\mathrm{km}, d_{10}$ the source distance in units of $10 \mathrm{kpc}$ and $\theta$ the inclination.

${ }^{b}$ Comptonized model parameters: $k T_{0}, k T_{\mathrm{e}}$ are resp. the seed photon and plasma temperature, $\tau$ the plasma optical depth for a disk geometry and $N_{\text {Comptt }}$ the normalization.

${ }^{c}$ Unabsorbed $0.01-300 \mathrm{keV}$ flux, which we take to be bolometric

${ }^{d}$ Average ASM count rate during the observation.

three instruments simultaneously with the generic LMXB model (see e.g. Sidoli et al. 2001), consisting of a multi-temperature disk black body (Mitsuda et al. 1984; Makishima et al. 1986; "diskbb" in XSPEC) in combination with a comptonized spectrum (Titarchuk 1994; Hua \& Titarchuk 1995; Titarchuk \& Lyubarskij 1995; "comptt" in XSPEC), both absorbed by cold interstellar matter following the model by Balucinska-Church \& McCammon (1992) ("phabs" in XSPEC). During the spectral analysis we allow the normalization between the three instruments to vary and find that the best fit values are within acceptable limits: the normalization between the LECS and MECS is $0.897 \pm 0.003$, while 0.7 to 1.0 is acceptable; between the PDS and MECS the normalization is $1.21 \pm 0.04$, while 1.1 to 1.3 is acceptable (Fiore et al. 1999). The results of the fit as well as the derived flux are provided in Table 1. The flux is determined by extrapolating the model from the observed range to $0.01-$ $300 \mathrm{keV}$. Extrapolating the model further while correcting for the interstellar absorption does not lead to a significant increase in flux. Under the assumption that no other features in the spectrum other than those we modeled have a substantial flux contribution, we refer to this as the bolometric flux. From the average ASM flux during this observation, we find that $1 \mathrm{ASM} \mathrm{c} \mathrm{s}^{-1}$ is equivalent to $(4.4 \pm 0.2) \times 10^{-10} \mathrm{erg} \mathrm{s}^{-1} \mathrm{~cm}^{-2}$.

To further study the bolometric correction that may be applied to the ASM data as a function of the persistent flux, we employ 160 observations carried out with the RXTE PCA between March 1996 and February 2006. The PCA spectra are extracted from the standard products, with exposure times between $96 \mathrm{~s}$ and $13 \mathrm{ks}$ and a total exposure of $310 \mathrm{ks}$. They are modeled between 3 and $40 \mathrm{keV}$ (the calibrated bandpass) with a generic model consisting of a black body and a cutoff power law, both absorbed following the model by Morrison \& McCammon (1983) with $N_{\mathrm{H}}=8 \times 10^{21} \mathrm{~cm}^{-2}$. The resulting fits are acceptable in 144 cases with $\chi_{\text {red }}^{2}<2$; in the remaining cases $\chi_{\text {red }}^{2}$ rises to up to 6. Despite the sometimes bad fit, we employ all observations since we are interested only in obtaining constraints for the bolometric correction and not in physical implications of the spectral

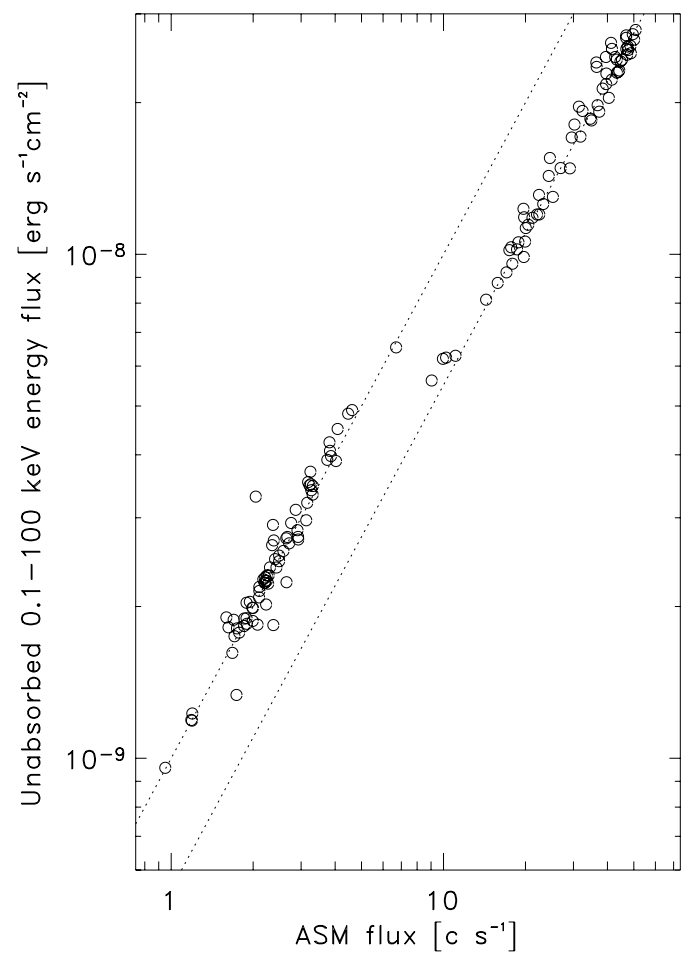

Fig. 2. Flux as a function of the ASM count rate. The distinction between the two spectral states is clearly visible. The two lines are not fits, but act as guides to distinguish the two states.

model. The fit outcome is tested leaving $N_{\mathrm{H}}$ free: no evidence is found for fluctuations in $N_{\mathrm{H}}$ above $5 \times 10^{22} \mathrm{~cm}^{-2}$. We determine the photon flux between 1.5 and $12 \mathrm{keV}$ before correcting for $\mathrm{ab}$ sorption and normalize it to observed ASM fluxes through calibration with Crab fluxes and by cross correlating the PCA measurements for 4U 1608-522 with contemporaneous ASM fluxes. We calculate the 0.1 to $100 \mathrm{keV}$ energy flux after a correction for absorption. Obviously, this implies a fairly large extrapolation from the 3 to $40 \mathrm{keV}$ PCA bandpass and we check how much flux is found outside that bandpass. It varies between $10 \%$ at the brightest state to $60 \%$ in the faintest state. This indicates what the maximum error is of the extrapolation. The results are plotted in Fig. 2. It clearly shows the two states identified by van Straaten et al. (2003). The boundary between both states is at 5 to 10 ASM c s${ }^{-1}$. At the bright state, when the spectrum is relatively soft, $1 \mathrm{ASM} \mathrm{c} \mathrm{s}^{-1}$ is equivalent to $5.5 \times 10^{-10} \mathrm{erg} \mathrm{s}^{-1} \mathrm{~cm}^{-2}$ and at the hard faint state to $1.0 \times 10^{-9} \mathrm{erg} \mathrm{s}^{-1} \mathrm{~cm}^{-2}$. For the bright state we can calibrate this result using the BeppoSAX broadband observation of the 1998 outburst from which we derived above the equivalence of $1 \mathrm{ASM} \mathrm{c} \mathrm{s}^{-1}$ to $(4.4 \pm 0.2) \times 10^{-10} \mathrm{erg} \mathrm{s}^{-1} \mathrm{~cm}^{-2}$. We use this number as conversion factor for the high flux state.

Applying the bolometric correction factors to the ASM flux, we find that the average persistent flux was $(2.313 \pm 0.013) \times$ $10^{-9} \mathrm{erg} \mathrm{cm}^{-2} \mathrm{~s}^{-1}$, while the peak flux reached during the three large outbursts was $2.2 \times 10^{-8}$ to $3.0 \times 10^{-8} \mathrm{erg} \mathrm{cm}^{-2} \mathrm{~s}^{-1}$. The mass accretion rate is on average $\dot{M} / \dot{M}_{\text {Edd }}=(1.8 \pm 0.2) \times 10^{-2}$ and 0.17 to 0.23 in the peaks of the outbursts.

Using the conversion factor of ASM counts to bolometric flux for count rates above $10 \mathrm{c} \mathrm{s}^{-1}$ we determine the fluence of the three major outbursts observed by the ASM for the duration that the count rate exceeded $20 \mathrm{c} \mathrm{s}^{-1}$. For the last outburst we also determined the fluence up to the moment that the superburst occurred. $20 \mathrm{c} \mathrm{s}^{-1}$ corresponds to an accretion rate of $\dot{M} / \dot{M}_{\text {Edd }}=(6.7 \pm 0.8) \times 10^{-2}$ and is chosen as a lower limit since 
Table 2. Outbursts properties. Between parentheses we give the $1 \sigma$ uncertainties in the least significant digit(s).

\begin{tabular}{lllllll}
\hline \hline Outburst & $\begin{array}{l}\text { Start time }^{a} \\
(\mathrm{MJD})\end{array}$ & $\begin{array}{l}\text { Duration }^{a} \\
(\mathrm{days})\end{array}$ & $\begin{array}{l}\text { Fluence } \\
\left(10^{-2} \mathrm{erg} \mathrm{cm}^{-2}\right)\end{array}$ & $\begin{array}{l}\text { Energy } \\
\left(10^{44} \mathrm{erg}\right)\end{array}$ & $\begin{array}{l}\text { Peak Flux } \\
\left(10^{-8} \mathrm{erg} \mathrm{s}^{-1} \mathrm{~cm}^{-2}\right)\end{array}$ & Peak $\dot{M} / \dot{M}_{\mathrm{Edd}^{d}}{ }^{d}$ \\
\hline 1 & 50848.0 & 44.0 & $6.3(3)$ & $0.77(15)$ & $3.0(2)$ & $0.23(3)$ \\
2 & 52475.0 & 48.0 & $6.9(3)$ & $0.85(16)$ & $2.6(2)$ & $0.20(3)$ \\
3 & 53437.5 & 79.5 & $10.4(5)$ & $1.3(2)$ & $2.24(19)$ & $0.17(2)$ \\
3 before SB & 53437.5 & 57.6 & $8.2(4)$ & $1.01(19)$ & $2.24(19)$ & $0.17(2)$ \\
\hline
\end{tabular}

${ }^{a}$ Start time and duration of time interval when the outburst flux exceeds $20 \mathrm{ASM} \mathrm{c} \mathrm{s}{ }^{-1}$.

${ }^{b}$ Energy calculated from fluence using distance $d=3.2 \pm 0.3 \mathrm{kpc}$.

${ }^{c}$ Unabsorbed bolometric peak flux determined from the 1-day ASM light curve $\left(10^{-8} \mathrm{erg} \mathrm{s}^{-1} \mathrm{~cm}^{-2}\right)$.

${ }^{d}$ Peak mass accretion rate in units of the Eddington limit assuming a $1.4 M_{\odot}$ neutron star.

it is close to the value of 0.1 that is required for the production of carbon (Cumming \& Bildsten 2001), while selecting the periods of high flux in one interval for each outburst. The bolometric fluences as well as the peak fluxes during the outbursts are presented in Table 2.

\subsection{Long-term bursting behavior}

Indicated in Fig. 1 are the bursts detected from 4U 1608-522 with the WFC on BeppoSAX until the mission ended in April 2002, the IBIS/ISGRI on INTEGRAL until September 2005 (Chelovekov et al. 2007), the PCA (Galloway et al. 2006) and the ASM on RXTE. One burst was detected by both the WFC and PCA. The ASM bursts are identified through 1) searching individual 90-s average dwell data of 4U 1608-522 for isolated high points that are at least $3 \sigma$ above a 100-day running average, resulting in roughly 200 burst candidates; 2) studying the raw 1 -s resolution light curves of the candidate dwells, to eliminate candidates that have no clear flare feature with a time scale smaller than the 90-s dwell time; 3) searching for flares with clear fastrise exponential-decay profiles, resulting in the identification of 7 certain bursts. The remaining 12 candidates are classified as tentative bursts from $4 \mathrm{U} 1608-522$.

For studying the bursting behavior as a function of the persistent flux, Fig. 3 shows a histogram of the ASM-measured persistent flux when a burst is seen for all bursts observed with the WFC, PCA and IBIS/ISGRI as well as the certain bursts observed with the ASM. There is the suggestion that bursts are rare during major outbursts while much more frequent during the extended low-intensity states during MJD 50 150-50 500 and 50 897-51 800. Quantitatively, however, the data are inconclusive in this respect. The PCA data set is somewhat biased, because those observations were targeted at 4U 1608-522 mainly when it was in outburst. The other data sets are serendipitous and, therefore, present an objective view of the bursting behavior. The WFC exposure time during major outbursts is 21.3 times smaller than during low-intensity states, while the ratio in the number of bursts is 1 to 36.2 out of 19 bursts observed by IBIS/ISGRI take place during a major outburst, while the source is observed in outburst during $12.7 \%$ of the exposure time. The ASM data are also inconclusive: 2 out of 19 bursts occurred at 1day average fluxes of $10 \mathrm{c} \mathrm{s}^{-1}$ while the fraction of the total time spent in that flux regime is $7.4 \%$. The WFC, PCA, IBIS/ISGRI and ASM light curves exhibit bursts with an average recurrence time of, respectively, 1.19, 0.60, 3.79 and 1.71 days.

The bursting behavior is characterized by $\alpha$ : the ratio of persistent fluence between bursts and the burst fluence. Using the PCA observations we calculate the average value of $\alpha$ both for the outbursts, when the ASM count rate was in excess of $20 \mathrm{c} \mathrm{s}^{-1}$,

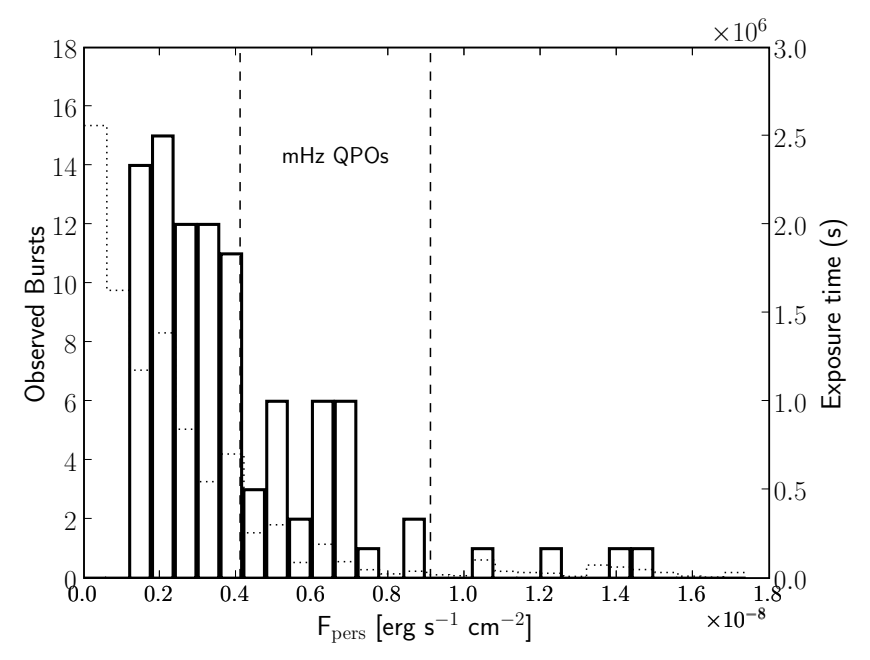

Fig. 3. Number of observed bursts (solid line) as a function of persistent flux $F_{\text {pers }}$. For the latter we use the average ASM count rate in a oneday interval around the moment the burst occurred converted to bolometric flux. This includes all bursts observed with BeppoSAX WFC, RXTE PCA, INTEGRAL IBIS/ISGRI as well as the clear bursts identified in the RXTE ASM light curve. The dashed lines indicate the region of $F_{\text {pers }}$ where Revnivtsev et al. (2001) observed mHz QPOs (see Sect. 5.4). The dotted line represents the total PCA, WFC, IBIS/ISGRI and ASM exposure time as a function of the ASM count rate. For PCA, WFC and IBIS/ISGRI observations we use the average ASM count rate during an observation. Together with the burst histogram, one observes that the burst rate does not vary significantly as a function of accretion rate in the range where type-I bursts occur.

and for all observations when the ASM count rate was below this level, but not during quiescence. Galloway et al. (2006) determined the fluence of 31 of the type-I X-ray bursts observed with the PCA. We find the persistent fluence between bursts by multiplying the average ASM flux during the PCA observations by the burst recurrence time. For the latter we take the ratio of the total PCA exposure time and the number of observed bursts during either the outbursts or the low-flux state. During the outbursts the PCA observed only one burst. Therefore, from the PCA data we only have a lower limit to the burst recurrence time in the outbursts, from which we find the lower limit $\alpha \geq 745 \pm 9$. The PCA observations when the ASM flux was less than $20 \mathrm{cs}^{-1}$ yield an average value of $\alpha=51.5 \pm 0.2$. 

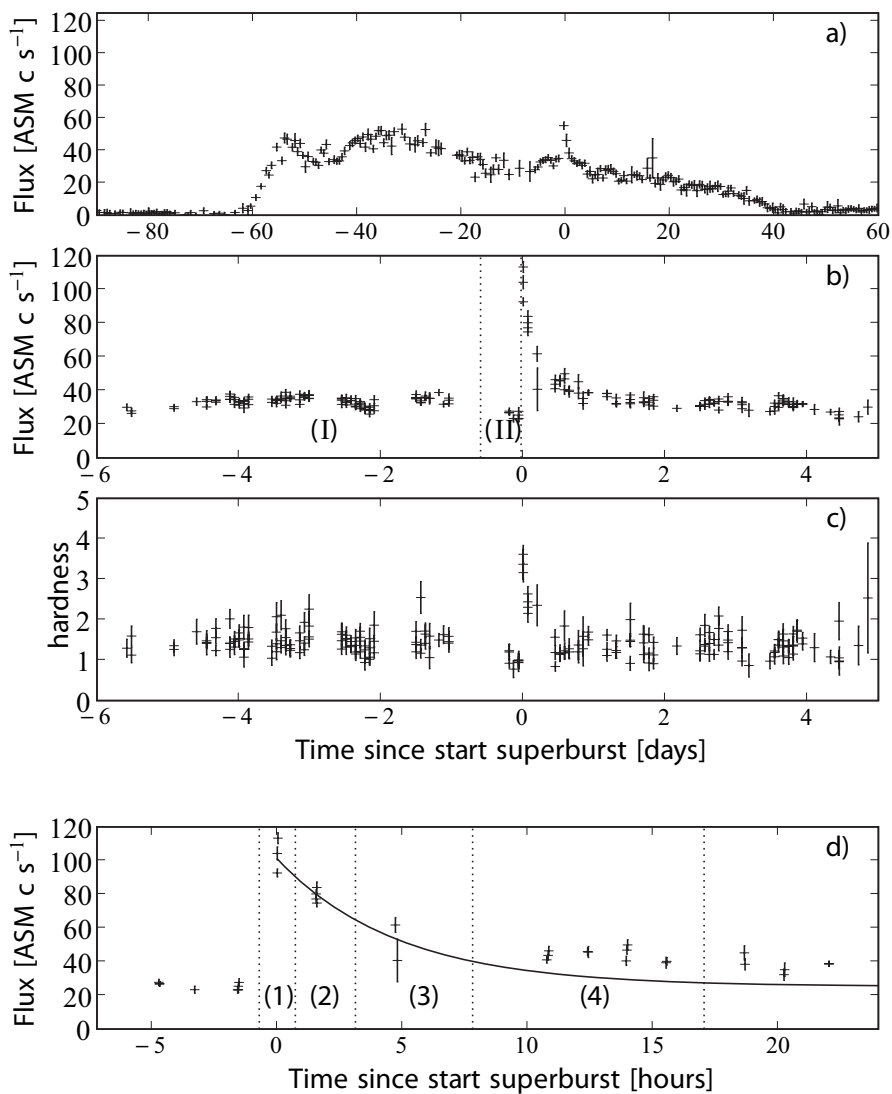

Fig. 4. Superburst light curves where each data point represents a $90 \mathrm{~s}$ dwell with its $1 \sigma$ uncertainty. a) $1.5-12 \mathrm{keV}$ ASM count rate at a 0.5 day time resolution. Shown is the outburst in which the superburst takes place. b) Zoomed-in part of $a$. Indicated are intervals I and II of the persistent flux prior to the burst. c) hardness ratio for each dwell, defined as the ratio of the counts in the $5-12 \mathrm{keV}$ and the $1.5-3 \mathrm{keV}$ energy bands. One data point with an error in excess of 4 is excluded from the plot. d) Zoomed-in part of top figure on the superburst. Burst intervals 1-4 are indicated as well as the exponential decay fitted to the first $5 \mathrm{~h}$ of the burst with an e-folding decay time of $4.8 \mathrm{~h}$ (see Table 3 ).

\section{Superburst analysis}

\subsection{Light curve}

In Fig. 4 we show the ASM light curve of 4U 1608-522 for the outburst in which the superburst takes place. The onset of the superburst is not observed by the ASM, as it falls in a $1.5 \mathrm{~h}$ data gap. Thirty-three minutes before the start of the superburst observation by the ASM, the light curves of the WXM and FREGATE onboard HETE-2 exhibit a fast rise of $3.6 \mathrm{~s}$ followed by a slow decay (Fig. 5). Since the event took place at the end of the observation, only one minute of the flare is recorded before the instruments are switched off. Nevertheless, the light curve suggests that the decay is much longer than for a normal type-I burst. The WXM detector image of the flare is consistent with the position of 4U 1608-522 if the spacecraft's attitude changed by $0.6^{\circ}$ since the middle of the orbit. A variation of the attitude of this size is not uncommon at the end of an orbit. The FREGATE has no positional information, but 4U 1608-522 was in the field of view at that time. From both instruments spectral information is available in four energy bands. Due to the low number of observed photons per energy band, this information is of limited use for the WXM. For the FREGATE, the flare is observed in the 6 to $40 \mathrm{keV}$ and 6 to $80 \mathrm{keV}$ bands and not in higher energy bands, which is consistent with a type-I X-ray
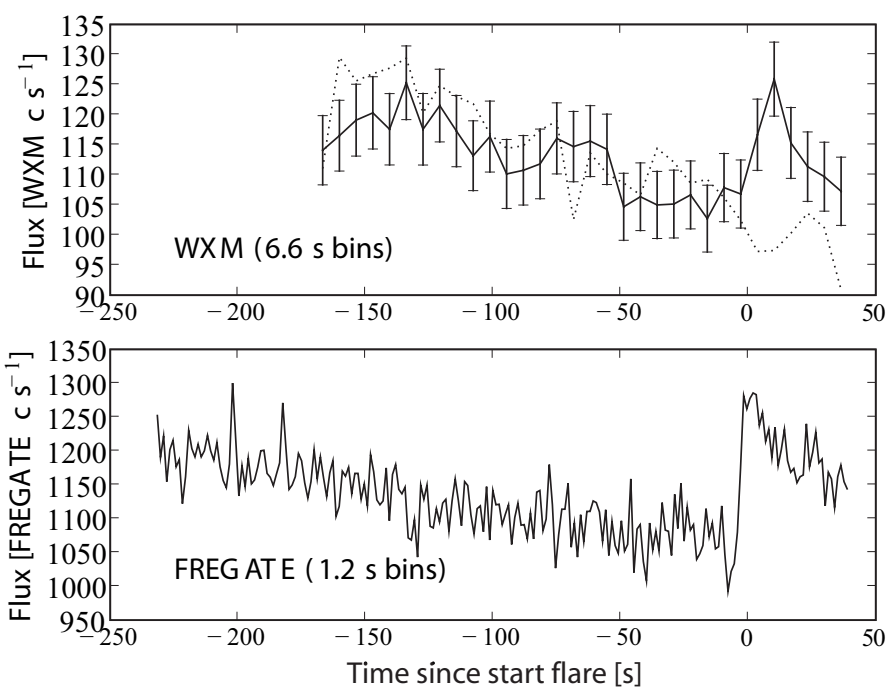

Fig. 5. HETE-2 light curve of likely onset superburst. Top: WXM. The data points show the count rate with $1 \sigma$ uncertainty observed by detector XB, while the dotted line indicates the count rate observed by detector XA. We employ data from the full $2-25 \mathrm{keV}$ band pass at a $6.6 \mathrm{~s}$ time resolution. The data is from the end of an observation, when the count rate decreases as the Earth covers an increasing part of the field of view. The flare is only visible in the XB light curve. From its position on the sky, $4 \mathrm{U} 1608-522$ was in the field of view of XB and not of XA. Bottom: FREGATE light curve from the $6-40 \mathrm{keV}$ band pass at $1.2 \mathrm{~s}$ time resolution.

burst (such as a superburst). Comparing this flare to a type-I X-ray burst from 4U 1608-522 which was previously observed with the WXM, the flare's peak height is approximately $80 \%$ of that of the burst. Therefore, the peak flux of the flare is at most $80 \%$ of the Eddington limit. As the time of occurrence, the source position and the peak flux are consistent with the superburst from $4 \mathrm{U}$ 1608-522, we regard this as a likely observation of the start of this superburst.

No precursor burst can be discerned. However, the presence of such a burst cannot be ruled out. If a potentially present precursor is similar to the type-I bursts observed during outbursts, it would have a short e-folding decay time of a few seconds and a peak flux of around $60 \%$ of the Eddington limit. Before the superburst onset the statistical quality of the FREGATE data is such that we can exclude at a $3 \sigma$ confidence level a precursor burst with a decay time of several seconds and a net peak flux of $\sim 10 \%$ of the Eddington limit or higher. However, the precursors observed from other hydrogen-accreting superbursters all took place at the superburst onset (Kuulkers et al. 2002b; Strohmayer \& Markwardt 2002; in't Zand et al. 2003). If the precursor and superburst light curves are superimposed, the presence of a precursor cannot be excluded from the data. Also, during the precursor burst the peak temperature might be lower than for the superburst, which would result in a lower observed flux in the FREGATE band. The WXM is sensitive down to lower energies, but the relative uncertainty in each data point is larger due to a smaller effective area, which may prevent us from detecting a precursor.

After the superburst took place, the first normal type-I X-ray burst was observed with IBIS/ISGRI after 99.8 days. Since there are frequent data gaps, whose durations are long with respect to the duration of an X-ray burst, 99.8 days is an upper limit to the burst quenching time.

In the $4.6 \mathrm{~h}$ preceding the superburst (interval II, see Fig. 4b) the persistent flux is at a level of $25.3 \mathrm{c} \mathrm{s}^{-1}$, while in the 7 days 
prior to that (interval I) it was $32.9 \mathrm{c} \mathrm{s}^{-1}$. The hardness ratio (Fig. 4c) indicates that the spectrum is harder in interval I. Approximately one day after the superburst peak, the count rate returns to the level of interval I. Only after 4.5 days does the count rate return to the level of interval II. This suggests that the superburst starts during a slight dip in the persistent flux. This makes it difficult to accurately separate the persistent emission from the burst emission during the decay. Therefore, we report results using both interval I and II.

The decay of superbursts is typically well-fit by an exponential. We fit the decay of the superburst with an exponential in all three channels separately as well as in the combined $1.5-12 \mathrm{keV}$ energy band (see Table 3 and Fig. 4d). In this fit we restrict ourselves to the first $5 \mathrm{~h}$ of the decay, to avoid the tail where an assessment of the persistent emission is difficult.

Not only is the count rate in interval II lower than in I. Also, the hardness ratio indicates that the spectrum is softer in interval II (see).

\subsection{Spectral analysis, energetics and layer thickness}

We fit the persistent emission ASM spectra from both interval I and II simultaneously with an absorbed power law, coupling the $N_{\mathrm{H}}$ value for both spectra. The best fit values as well as the 1.5-12 keV flux in each interval are provided in Table 3.

We divide the superburst in four time intervals, as indicated in Fig. 4d, from the part of the burst where most data points lie at $>3 \sigma$ above the persistent flux from interval II (when using interval I, we restrict ourselves to the first three burst intervals). These intervals contain in total 19 measurements. Note that some of these start at the same time, but are from different SSCs. A systematic error of 3\%, derived from the scatter in the Crab intensity (Levine et al. 1996), is included in the uncertainties of the data points. Levine et al. (1996) note that in many cases this is an underestimate of the systematic uncertainties. For each interval we extract a spectrum. We obtain the net-superburst spectrum by subtracting the persistent spectrum based on either interval I or II from the observed spectrum (see Kuulkers et al. 2002a, for a discussion on decoupling persistent and burst emission for normal type-I bursts). This net spectrum is fit with an absorbed black body.

During the spectral analysis we fix the hydrogen absorption column density. From BeppoSAX broad-band observations during outburst we find a hydrogen absorption column density of $N_{\mathrm{H}}=0.89 \times 10^{22} \mathrm{~cm}^{-2}$ (see Sect. 3.2). However, here we use the value of $N_{\mathrm{H}}=0.61 \times 10^{22} \mathrm{~cm}^{-2}$ which we obtained from the persistent emission in interval II, since we perform the superburst spectral analysis using similar ASM data and the same response matrix.

In the spectral analysis, our best fit has a reduced $\chi_{\text {red }}^{2}$ value of 3.5. We increased the uncertainties in the data points such that we obtained a fit with $\chi^{2}=1$. Using this fit we determined the uncertainties in the fitted parameters using $\Delta \chi^{2}=1$. Increasing the uncertainties to satisfy the "goodness of fit" condition $\chi_{\text {red }}^{2}$ was also required when we analyzed the Crab spectrum in Sect. 2.2. In this procedure the best fit values of the model parameters deviate from the previous best fit values by at most $1.37 \sigma$. We fit the four intervals simultaneously, keeping the value of the black body normalization the same for all spectra. Since the normalization parameter depends on the emission region size of the black body, this implies that the size is assumed not to change during the superburst decay. This is a valid approach, as we will see that the peak flux of the superburst is below the Eddington flux at which photospheric
Table 3. Superburst properties using persistent interval I and II with $1 \sigma$ uncertainties for the last digits between parentheses.

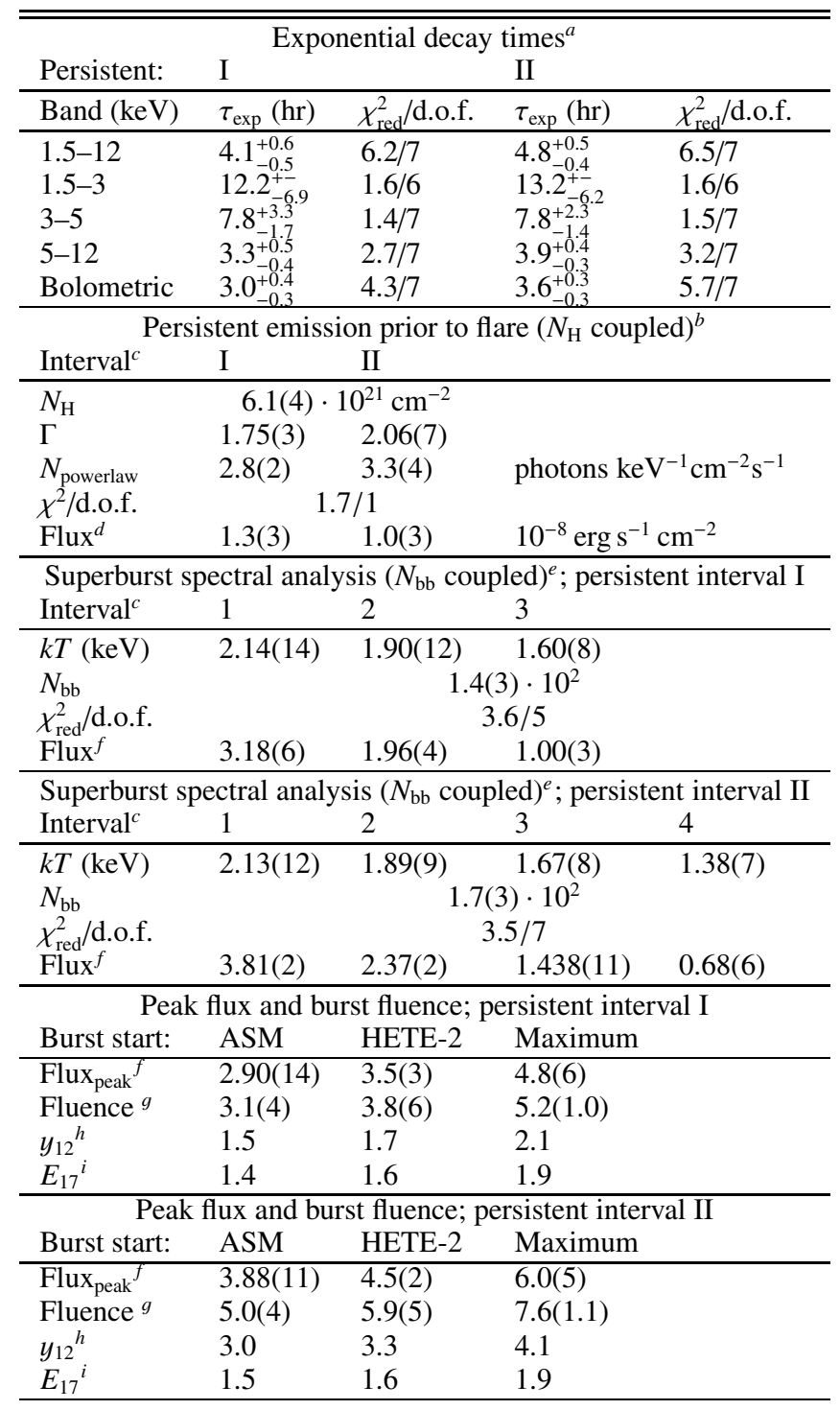

${ }^{a}$ In the $1.5-3 \mathrm{keV}$ band we find the decay to be consistent with a horizontal line, due to the large uncertainty of the data points in the tail of our fit interval.

${ }^{b}$ Absorbed power law model $\left(N_{\text {powerlaw }} E^{-\Gamma}\right)$ parameters: $N_{\mathrm{H}}$ the interstellar column density, $\Gamma$ the photon index and $N_{\text {powerlaw }}$ the normalization defined as photons $\mathrm{keV}^{-1} \mathrm{~cm}^{-2} \mathrm{~s}^{-1}$ at $1 \mathrm{keV}$.

${ }^{c}$ See Fig. $4 \mathrm{~b}$.

${ }^{d}$ Unabsorbed flux in $1.5-12 \mathrm{keV}$ energy range $\left(10^{-8} \mathrm{erg} \mathrm{s}^{-1} \mathrm{~cm}^{-2}\right)$.

${ }^{e}$ Black body model parameters: temperature $k T$ and normalization $N_{\mathrm{bb}} \equiv R^{2} / d^{2}$, with $R$ the source radius in $\mathrm{km}$ and d the source distance in $10 \mathrm{kpc}$.

${ }^{f}$ Unabsorbed bolometric flux $\left(10^{-8} \mathrm{erg} \mathrm{s}^{-1} \mathrm{~cm}^{-2}\right)$.

${ }^{g}$ Unabsorbed bolometric fluence $\left(10^{-4} \mathrm{erg} \mathrm{cm}^{-2}\right)$.

${ }^{h}$ Column depth $\left(10^{12} \mathrm{~g} \mathrm{~cm}^{-2}\right)$.

${ }^{i}$ Energy release per unit mass $\left(10^{17} \mathrm{erg} \mathrm{g}^{-1}\right)$.

expansion is expected. Fits where we did not use this coupling did not indicate a significant change in radius from one interval to the next. We provide the best fit values of the model parameters in Table 3. Assuming isotropic radiation and using the distance of $3.2 \pm 0.3 \mathrm{kpc}$ as well as the best fit value for the black 
body normalization, we find an emission region size equivalent to a sphere with a radius of $R=4.2 \pm 0.5 \mathrm{~km}$, which is consistent with the black body radii both Murakami et al. (1980) and Galloway et al. (2006) found for normal type I X-ray bursts.

To investigate the influence of the choice of $N_{\mathrm{H}}$ on the fit results, we repeated the spectral analysis using $N_{\mathrm{H}}=8.91 \times 10^{21} \mathrm{~cm}^{-2}$ (see Table 1 ). The difference in best fit values of the model parameters to those in the previous analysis never exceeds $1 \sigma$. The derived flux differs by at most $3 \%$.

Using the average count rate and the flux we obtained for each burst interval, we convert the count rate of each data point to flux. We fit an exponential function to the first $5 \mathrm{~h}$ of the resulting curve and determine the peak flux at the start of this interval. We find a peak flux of $22 \pm 3 \%(29 \pm 3 \%)$ of the Eddington flux using the persistent level from interval I (II). Integrating this exponential gives us the burst fluence, which at a distance of $3.2 \pm 0.3 \mathrm{kpc}$ corresponds to a burst energy of $(3.8 \pm 0.9) \times 10^{41} \mathrm{erg}\left((6.1 \pm 1.2) \times 10^{41} \mathrm{erg}\right)$. By extrapolating the exponential to the start of the flare observed by HETE-2, a peak flux of $27 \pm 4 \%(34 \pm 4 \%)$ of the Eddington flux and a burst fluence of $(4.7 \pm 0.8) \times 10^{41} \mathrm{erg}\left((7.2 \pm 0.9) \times 10^{41} \mathrm{erg}\right)$ are obtained. Extrapolating to the start of the ASM data gap preceding the observed part of the burst, we find an upper limit to the peak flux and burst fluence: respectively $36 \pm 6 \%(45 \pm 4 \%)$ of the Eddington flux and $(6 \pm 2) \times 10^{41} \mathrm{erg}\left((9 \pm 2) \times 10^{41} \mathrm{erg}\right)$ (see Table 3).

Cumming \& Macbeth (2004) present multizone numerical models for the cooling of the neutron star surface layers during the decay of a superburst as well as analytic fits to these models. The models depend on the ignition column depth $y \equiv$ $y_{12} 10^{12} \mathrm{~g} \mathrm{~cm}^{-2}$ and the energy release per gram $E \equiv E_{17} \times$ $10^{17} \mathrm{erg} \mathrm{g}^{-1}$. Cumming et al. (2006) fit these models to the light curves of six superbursts. We perform the same fit to the light curve of the superburst from $4 \mathrm{U} 1608-522$ using the persistent level from both interval I and II (Fig. 6). We find, respectively, $y_{12}=1.5, E_{17}=1.4$ and $y_{12}=3.0, E_{17}=1.5$. To estimate the values of $y_{12}$ and $E_{17}$ in case the superburst started at either the start of the flare observed with HETE-2 or at the start of the data gap in the ASM light curve, we use the scaling laws found by Cumming et al. (2006). Thus we obtain the ranges $y_{12}=1.5-2.1$, $E_{17}=1.4-1.9$ and $y_{12}=3.0-4.1, E_{17}=1.5-1.9$, using respectively the persistent level from interval I and II (see Table 3).

\section{Discussion}

The first detection of a superburst from a classical LMXB transient poses significant challenges to superburst theory and underlines shortcomings thereof that were already realized earlier. This shows the need for additional ingredients in the models. While the superburst characteristics of 4U 1608-522 are similar to those from other superbursters (see Table 4), the accretion rate on a time scale of ten years is a factor of 3 smaller than observed for the slowest accretor among all other superbursting sources.

In the following subsections we will see that there are two challenges: the destruction of carbon by the ever-present hydrogen/helium flashes is apparently not as important as theory predicts and the predicted crust temperatures fall short by a factor of 2 of the value required for the ignition of unstable carbon burning. Additionally, we make a few remarks on the $\mathrm{mHz}$ QPO observed in $4 \mathrm{U} 1608-522$.

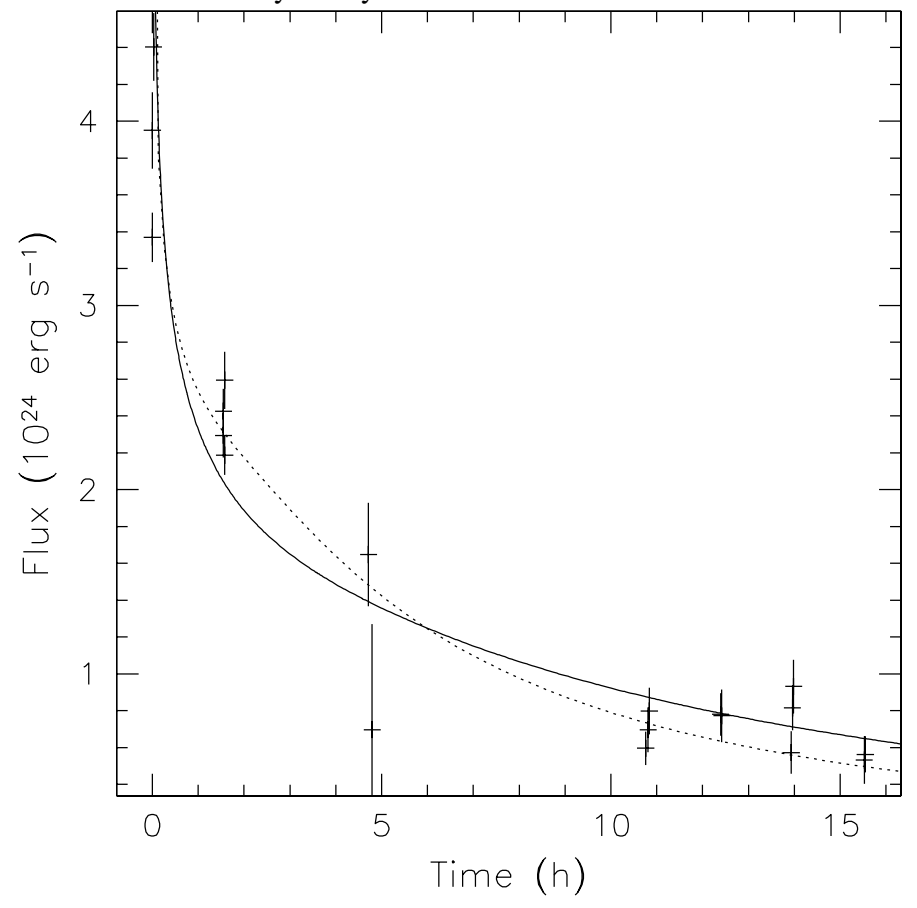

Fig. 6. Fit of cooling models to superburst decay. The data points are ASM count rates converted to flux for each ASM measurement (see Sect. 4.2) with $1 \sigma$ uncertainties. The persistent level from interval II is used (see Fig. 4b). The solid and dotted line represent fits with, respectively, the numerical and analytic cooling models from Cumming \& Macbeth (2004).

Table 4. Characteristics of the $4 \mathrm{U}$ 1608-522 superburst compared to 13 other superbursts (from Kuulkers 2004; and in't Zand et al. 2004). $\tau_{\exp }$ from fit to count rates converted to unabsorbed bolometric flux.

\begin{tabular}{lll}
\hline \hline & $4 \mathrm{U} 1608-522$ & Other superbursts \\
Duration $(\mathrm{h})$ & $5-22$ & $2-15$ \\
Precursor & No & Always when data available \\
$\tau_{\text {exp }}(\mathrm{h})^{a}$ & $\sim 4.5$ & $0.7-6$ \\
$k T_{\text {peak }}(\mathrm{keV})$ & $2.1 \pm 0.1$ & $1.8-3$ \\
$L_{\text {peak }}\left(10^{38} \mathrm{erg} \mathrm{s}^{-1}\right)$ & $0.4-0.7$ & $0.4-3.4$ \\
$L_{\text {pers }}\left(L_{\text {Edd }}\right)$ & $0.11-0.15$ & $0.1-0.25^{b}$ \\
$\left\langle L_{\text {pers }}\right\rangle\left(L_{\text {Edd }}\right)$ & 0.03 & $0.11-0.43^{c}$ \\
$E_{\mathrm{b}}\left(10^{42} \mathrm{erg}\right)$ & $0.4-0.9$ & $0.3-1.4$ \\
$\tau \equiv E_{\mathrm{b}} / L_{\text {peak }}(\mathrm{hr})$ & $1.0-1.3$ & $1.1-6.9$ \\
$\gamma \equiv L_{\text {pers }} / L_{\text {peak }}$ & $0.2-0.5$ & $0.1-0.7$ \\
$t_{\text {no bursts }}(\mathrm{d})$ & $<99.8$ & $>7.5$ \\
Donor & $\mathrm{H} / \mathrm{He}$ & $\mathrm{He}$ and $\mathrm{H} / \mathrm{He}$ \\
\hline
\end{tabular}

${ }^{a}$ Exponential decay time in $1.5-12 \mathrm{keV}$ band for $4 \mathrm{U} 1608-522$ and in similar energy bands for other superbursters.

${ }^{b}$ Persistent luminosity at the time of the superburst, excluding the candidate ultracompact source $4 \mathrm{U} 0614+091$ and the Z-source GX 17+2 which have a luminosity close to, respectively, 1\% (Kuulkers 2005; in 't Zand et al. 2007) and 100\% (in't Zand et al. 2004) of the Eddington luminosity.

${ }^{c}$ Average persistent luminosity from all ASM observations, again excluding 4U 0614+091 and GX 17+2.

\subsection{Recurrence time}

From fits of cooling models to the superburst light curve we find that the superbursting layer had at ignition a column depth of $y_{12}=1.5-4.1$ and an energy release per unit mass of $E_{17}=1.5-1.9$, which implies a total energy release of (2.6$9.8) \times 10^{42} \mathrm{erg}$. From the X-ray flux we derived an energy release of $(0.4-0.9) \times 10^{42} \mathrm{erg}$, which is only $5-45 \%$ of the total 


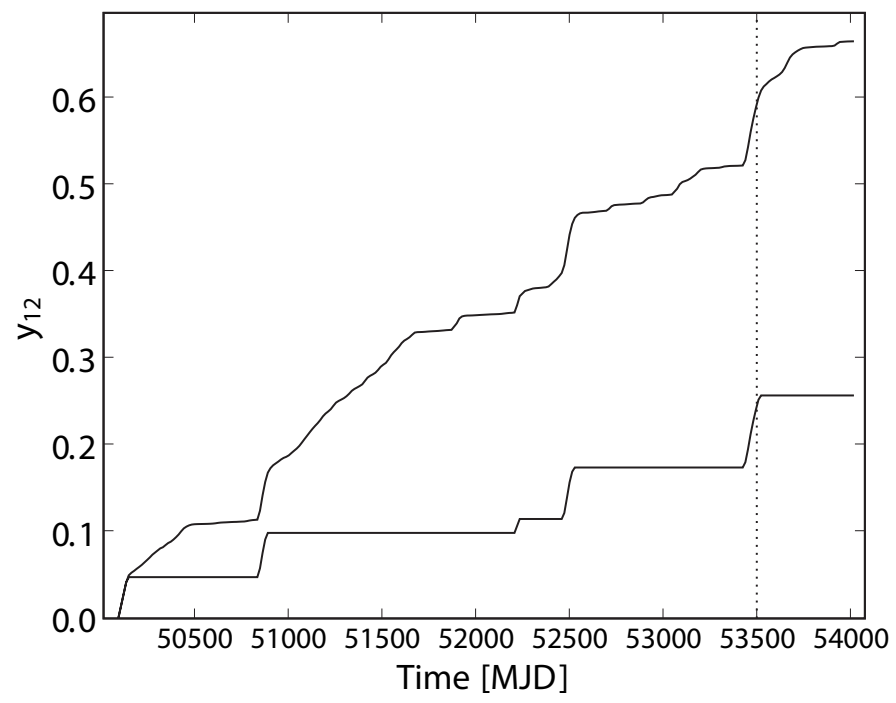

Fig. 7. Accreted column depth $y_{12} \equiv y /\left(10^{12} \mathrm{~g} \mathrm{~cm}^{-2}\right)$ over time. Assuming a neutron star of $1.4 M_{\odot}$ mass and $10 \mathrm{~km}$ radius at a distance of $3.2 \mathrm{kpc}$, we show the cumulative column depth that is accreted since the start of RXTE ASM observations as inferred from the X-ray flux at 14 day time resolution (cf. Fig. 1). The lower curve takes into account only matter that is accreted when $4 \mathrm{U} 1608-522$ was in the high state when the ASM count rate exceeded $20 \mathrm{c} \mathrm{s}^{-1}$, while the upper curve includes the low state as well. The dotted line indicates the moment the superburst was observed.

inferred from the cooling models (after correction for the gravitational redshift with a typical value of $1+z=1.3$ ). The rest of the energy is either released as neutrinos or is conducted to greater depths and radiated away on a longer timescale. Figure 7 shows the column depth that is observed to be accumulated since the start of the ASM observations. At the moment of the superburst a column of $y_{12}=0.55\left(y_{12}=0.27\right.$ taking into account only the stable burning in the high state) had been accreted over a period of 9.3 years. Note that this is derived under the assumption that the X-ray flux is a good measure for the mass accretion rate, which may not be the case (see discussion in Sect. 3.2). In fact, we assume that stable burning occurs when the ASM flux is in excess of $20 \mathrm{c} \mathrm{s}^{-1}$, which introduces an uncertainty of several tens of percents in the column depth. With an average accretion rate of $\dot{M}=(1.8 \pm 0.2) \times 10^{-2} \dot{M}_{\text {Edd }}$ the required column depth is accreted over a period of 26 to $72 \mathrm{yr}$. If carbon is only produced from matter accreted during stable burning in the high state, the recurrence time is twice as long. In both cases it is much longer than the 57.6 day duration of the 2005 outburst before the superburst occurred. This implies that the carbon fuel has been produced during multiple outbursts.

\subsection{Producing and preserving carbon}

Schatz et al. (2003) found from nucleosynthesis calculations that carbon is produced most efficiently through the stable nuclear burning of hydrogen and helium. Unstable burning during type-I $\mathrm{X}$-ray bursts results in significantly less carbon $(\lesssim 1 \%$ by mass; Woosley et al. 2004), but produces heavier ashes. Cumming \& Bildsten (2001) proposed that these heavy ashes are important because they set the opacity and therefore temperature of the superbursting layer, although Brown (2000) later showed that this effect is much smaller than initially estimated once the temperature profile of the entire crust was taken into account. Therefore, it is interesting to ask whether both burning regimes are necessary for producing the fuel for superbursts. Certainly, all known superbursters exhibit both type-I X-ray bursts and a high $\alpha$ value, which are indications for both unstable and stable burning, respectively.

The stable burning of helium takes place at accretion rates $\dot{M}$ in excess of $10 \%$ of Eddington. Therefore, we expect that in $4 \mathrm{U} 1608-522$ carbon is created during the outbursts. This carbon could be destroyed by type-I X-ray bursts, which are observed during all flux states. For carbon to survive the bursts, it has to reside in a layer which is depleted of hydrogen. Woosley et al. (2004) find this to be the case at the bottom of a bursting layer. The different composition of the ashes of unstable hydrogen/helium burning as opposed to stable hydrogen/helium burning (Woosley et al. 2004; Schatz et al. 2003) will likely mix rapidly. If a period of stable burning was followed by unstable burning, the heavier ashes of the latter would rapidly undergo a Rayleigh-Taylor instability and mix with the lighter ashes below.

An important question is whether the carbon is able to burn stably during the low state. Cumming \& Bildsten (2001) argued that carbon would burn stably at low accretion rates, and suggested that this may explain why superburst sources have accretion rates of $\approx 0.1$ Eddington and up. This question is crucial to understand for $4 \mathrm{U} 1608-522$, since the accretion rate is low during quiescence and the low persistent state. Further modelling of the production and destruction of carbon during the cycle of transient outbursts is needed.

A complication here is that at the low accretion rates that occur during quiescence and in the low persistent state in 4U 1608522 , the accretion may be slow enough that the carbon and heavy elements in the ashes of normal type-I bursts gravitationally separate, with the carbon floating upwards relative to the heavy elements. To estimate the relative drift velocity of a carbon nucleus in the heavy element ocean, we follow the work of Bildsten \& Hall (2001), who estimated the rate of sedimentation of ${ }^{22} \mathrm{Ne}$ in the interiors of $\mathrm{CO}$ white dwarfs. They start with the self-diffusion coefficient for a one component plasma, $D \approx 3 \omega_{\mathrm{p}} a^{2} \Gamma^{-4 / 3}$ (Hansen et al. 1975), where $\omega_{\mathrm{p}}$ is the ion plasma frequency, $a$ is the inter-ion separation, and $\Gamma$ is the standard parameter that measures the strength of the Coulomb interactions between ions. A single carbon nucleus in a background of ${ }^{56} \mathrm{Fe}$ feels an upward force of $12 m_{\mathrm{p}} g / 13$, giving a drift velocity $v=54 m_{\mathrm{p}} g / 169 \mathrm{e} \Gamma^{1 / 3}(4 \pi \rho)^{1 / 2}$ (compare Bildsten \& Hall 2001 Eq. [3]). Writing the time to cross a pressure scale height at this drift velocity as $t_{\mathrm{s}}=H / v=P / \rho g v$, and assuming that the pressure is provided by degenerate relativistic electrons (a good approximation at these depths), we find

$t_{\mathrm{s}}=7.2$ years $y_{12}^{13 / 24}\left(\frac{T_{8}}{6}\right)^{-1 / 3}\left(\frac{g_{14}}{2.45}\right)^{-31 / 24}$

where we assume that the heavy element is ${ }^{56} \mathrm{Fe}$ (the scaling is $t_{\mathrm{s}} \propto Z^{37 / 18} / A$ ). This timescale is equal to the accretion timescale $t_{\text {accr }}$ for $\dot{m} \approx 0.05 \dot{m}_{\text {Edd }}$. The sedimentation timescale $t_{\mathrm{s}}$ is comparable to the timescale on which we infer the carbon fuel is accumulated, so that the physics of the relative separation of the carbon and heavy elements should be considered further.

\subsection{The crust temperature and carbon ignition}

Although the accretion rate of $4 U$ 1608-522 reaches $\dot{M} \lesssim 0.23 \dot{M}_{\text {Edd }}$ during the peak of the outburst, the high accretion rate only lasts a short $(\$ 100 \mathrm{~d})$ time, so that the crust does not reach a thermal steady-state. The temperature of the 


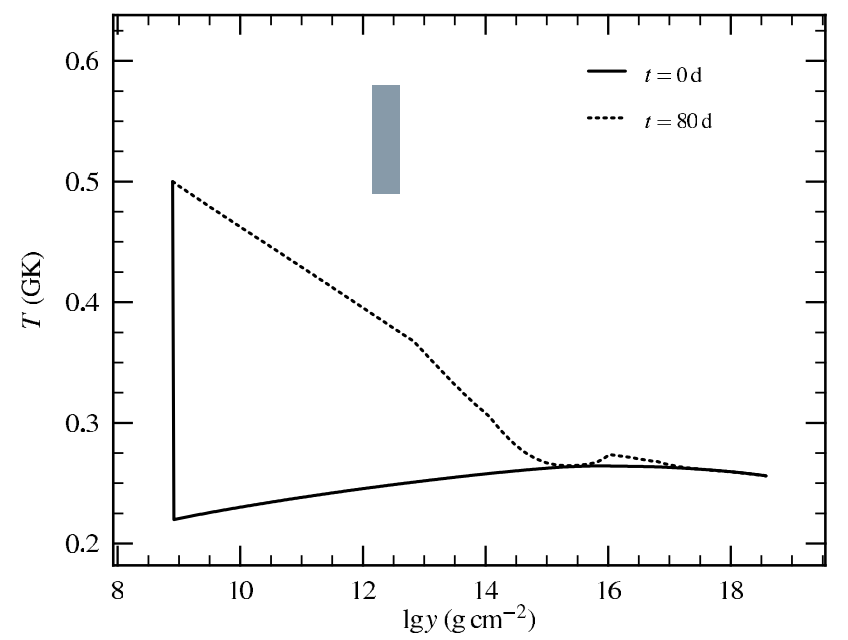

Fig. 8. Temperature evolution in the crust of $4 \mathrm{U} 1608-522$ during the outburst. At the beginning of the outburst (solid line) we set the temperature at a column $y=10^{9} \mathrm{~g} \mathrm{~cm}^{-2}$ to $5 \times 10^{8} \mathrm{~K}$. After 80 days, the temperature in the outer crust has risen (dotted line), but is still well below the temperature for which a one-zone stability analysis would predict a superburst with a depth inferred from fits to the cooling (shaded box).

deep crust can be inferred by observations of the quiescent luminosity (Rutledge et al. 1999). Hence this source provides a test of the strength and location of heat sources in the crust. The question is whether the crust can reach temperatures sufficiently hot to initiate thermally unstable ${ }^{12} \mathrm{C}$ fusion in the short time available during the outburst. To check this, we constructed a time-dependent model based on recent calculations of the heating from electron capture reactions in the crust (Gupta et al. 2007). We set the mass number to $A=40$, which yields a large heat release $(0.40 \mathrm{MeV}$ per accreted nucleon $)$ in the outer crust. For the inner crust, we use the model of Haensel $\&$ Zdunik (1990). We integrated the thermal diffusion equation using a standard method-of-lines formalism. The microphysics is identical to that in Gupta et al. (2007). The total heat released in the crust during outburst is $1.8 \mathrm{MeV} \mathrm{u}^{-1}$.

As an initial condition, we computed the temperature for steady accretion at the time-averaged mass accretion rate $\langle M\rangle=$ $6.5 \times 10^{15} \mathrm{~g} \mathrm{~s}^{-1}$. We then ran through a series of 60 outburst/quiescent cycles, to ensure that the crust had reached a limit cycle. During outburst we set $\dot{M}=1.3 \times 10^{17} \mathrm{~g} \mathrm{~s}^{-1}$, with a duration of $80 \mathrm{~d}$; the quiescent interval was set to $4.16 \mathrm{yr}$. During quiescence we used a relation between the temperature at a column $y=10^{9} \mathrm{~g} \mathrm{~cm}^{-2}$ and the surface luminosity appropriate for an accreted envelope (Brown et al. 2002). The quiescent luminosity reached a minimum of $L_{\mathrm{q}}=6.1 \times 10^{33} \mathrm{erg} \mathrm{s}^{-1}$, in reasonable agreement with the value derived by Rutledge et al. (2000). Figure 8 shows the change in temperature, as a function of column, during the outburst. At the start of the outburst (solid line), we set the temperature at a column $y=10^{9} \mathrm{~g} \mathrm{~cm}^{-2}$ to $5 \times 10^{8} \mathrm{~K}$. This is a reasonable upper limit on the temperature set by steady-state $\mathrm{H} / \mathrm{He}$ burning. After $80 \mathrm{~d}$, the temperature in the outer crust has risen substantially (dotted line), but is still much cooler than needed to explain the fitted ignition column and temperature (shaded box).

We conclude that heating in the crust from electron captures, neutron emissions, and pycnonuclear reactions, is insufficient to raise the crust temperature during the outburst enough for ${ }^{12} \mathrm{C}$ to ignite at the inferred column.
In the other superburst sources, which are either persistent accretors, or transients with long duration outbursts (e.g. KS 1731-260), the crust reaches a thermal steady state. Even in these sources, theoretical models have difficulty explaining the inferred superburst ignition columns of $\sim 10^{12} \mathrm{~g} \mathrm{~cm}^{-2}$ (Brown 2004; Cooper \& Narayan 2005; Cumming et al. 2006; Gupta et al. 2007). The properties of the neutron star crust and core that affect the thermal profile such as neutrino emissivity and crust conductivity must all be chosen to maximize the crust temperature in order to achieve the observed ignition depths. In fact, Cumming et al. (2006) showed that it was impossible to reproduce the observations if neutrino cooling due to Cooper pair formation of superfluid neutrons in the crust (e.g. Yakovlev et al. 1999) was included, because this efficient neutrino emission mechanism led to an upper limit on the crust temperature at the ignition depth of $\approx 4 \times 10^{8} \mathrm{~K}$, less than the temperature of $\approx 6 \times 10^{8} \mathrm{~K}$ required for carbon ignition. Recent work by Leinson $\&$ Pérez (2006) has shown that the neutrino emission due to this mechanism has been overestimated in previous calculations, and that this mechanism is in fact not the dominant source of neutrino emissivity.

As we have shown, the short duration of the transient outbursts in $4 \mathrm{U} 1608-522$ means that the crust is heated to much lower temperatures than those corresponding to thermal equilibrium. Therefore the observation of a superburst from $4 \mathrm{U} 1608-522$ is deeply puzzling and presents a significant challenge to current superburst models.

\subsection{Bursting behavior and $\mathrm{mHz}$ QPOs}

The normal type-I X-ray bursting behavior looks similar to what is seen in persistent bursting sources with strongly variable persistent fluxes, most notably 4U 1820-303 (Chou \& Grindlay 2001), GX 3+1 (den Hartog et al. 2003), 4U 1705-44 and KS 1731-260 (Cornelisse et al. 2003). In those cases X-ray bursts become far less frequent or even absent during high flux states. Also in the case of $4 \mathrm{U} 1608-522$ the bursting behavior is seen to differ between the low and high flux state. Murakami et al. (1980) report that in the high flux state bursts have a shorter duration and a higher $\alpha$ value than the bursts in the low flux state, while the burst rate is not significantly different. The bursts observed with the RXTE PCA and the BeppoSAX WFCs exhibit this behavior as well. This would be consistent with hydrogen and helium burning becoming predominantly stable at higher mass accretion rates (van Paradijs et al. 1988).

Revnivtsev et al. (2001) examined EXOSAT and RXTE PCA data of 4U 1608-522 and discovered low frequency quasiperiodic oscillations ( $\mathrm{mHz}$ QPOs). They observed no type-I $\mathrm{X}$-ray bursts when the persistent emission was higher than when the QPOs were detected, which supported the hypothesis that $\mathrm{mHz}$ QPOs occur at the transition of unstable to stable burning, such that at higher accretion rates the bursting behavior ceases (see also Heger et al. 2007). Interestingly, the PCA, WFCs and IBIS/ISGRI have observed a total of four X-ray bursts when the persistent flux was up to a factor of two higher than when $\mathrm{mHz}$ QPOs were seen (see Fig. 3). Although this disagrees with the prediction of the marginally stable burning model of Heger et al. (2007), the hypothesis could still be valid if, as discussed by Heger et al. (2007), the conditions for nuclear burning vary substantially across the neutron star surface, such that on one part of the surface unstable burning gives rise to type-I bursts while on another part $\mathrm{mHz}$ QPOs occur. The scenario that ignition conditions might vary across the surface of the star for rapidly rotating neutron stars has been discussed by Cooper \& Narayan (2007; 
see also Spitkovsky et al. 2002), although the predicted range for the global accretion rate where both stable and unstable burning take place at different latitudes is a factor of 3 higher than where $\mathrm{mHz}$ QPOs are observed from 4U 1608-522.

\section{Summary and conclusions}

For the first time a superburst has been observed from a transiently accreting neutron star in a low-mass X-ray binary where the duration of the accretion outbursts is short with respect to the expected superburst recurrence time. 4U 1608-522 exhibited a superburst 55 days after the onset of an accretion outburst. We analyzed the superburst as well as the long term accretion and bursting behavior of $4 \mathrm{U} 1608-522$. We found the properties of the superburst to be comparable to those of the long superbursts observed from 4U 1254-690 and KS 1731-260. Fits of cooling models to the superburst light curve indicate that the superburst ignited when a column of $y=(1.5-4.1) \times 10^{12} \mathrm{~g} \mathrm{~cm}^{-2}$ had been accreted. From the average X-ray flux that the RXTE ASM observed from $4 \mathrm{U} 1608-522$ over the past 11 years, we derive that this column was accreted over a period of 26 to 72 years.

The difficulties that superburst theory has in explaining the ignition column depths derived for other superbursters, are underlined by the observation of the superburst in 4U 1608-522. The transient nature of the accretion poses challenges for superburst models. The average accretion rate over the past 11 years was much lower than for the other superbursters, with the possible exception of 4U 0614+091 (Kuulkers 2005). At the derived column depth, current superburst theory predicts that during the timespan of the outbursts the crust temperature does not rise to the value required for superburst ignition. Furthermore, the carbon that fuels the superburst is expected to be produced only during the outbursts, while the stable nuclear burning of ${ }^{12} \mathrm{C}$ as well as the frequent type-I X-ray bursts lower the carbon abundance. The detection of the superburst implies that a significant amount of carbon survives the long periods outside the outbursts. The balance between the creation and destruction processes is influenced by mixing and sedimentation in the neutron star envelope. The effect of these requires further study before predictions about the produced amount of superburst fuel can be made.

Acknowledgements. L.K. acknowledges support from The Netherlands Organization for Scientific Research (NWO). Nuovo Telespazio and the BeppoSAX Science Data Center are thanked for continued support. This research made use of the RXTE/ASM database. A.C. is currently supported by an NSERC Discovery Grant, Le Fonds Québécois de la Recherche sur la Nature et les Technologies, and the Canadian Institute for Advanced Research, and is an Alfred P. Sloan Research Fellow. E.F.B. acknowledges support by Chandra Award Number TM7-8003X issued by the Chandra X-ray Observatory Center, which is operated by the Smithsonian Astrophysical Observatory for and on behalf of the National Aeronautics Space Administration under contract NAS8-03060. A.C. and E.F.B. thank the Institute for Nuclear Theory at the University of Washington for its hospitality and the Department of Energy for partial support during the completion of this work.

\section{References}

Arnaud, K. A. 1996, in Astronomical Data Analysis Software and Systems V, ed. G. H. Jacoby \& J. Barnes, ASP Conf. Ser., 101, 17

Atteia, J.-L., Boer, M., Cotin, F., et al. 2003, in American Institute of Physics Conference Series, Gamma-Ray Burst and Afterglow Astronomy 2001: A Workshop Celebrating the First Year of the HETE Mission, ed. G. R. Ricker, \& R. K. Vanderspek, 662, 17

Balucinska-Church, M., \& McCammon, D. 1992, ApJ, 400, 699

Belian, R. D., Conner, J. P., \& Evans, W. D. 1976, ApJ, 206, L135

Bildsten, L., \& Hall, D. M. 2001, ApJ, 549, L219
Boella, G., Butler, R. C., Perola, G. C., et al. 1997a, A\&A, 122, 299

Boella, G., Chiappetti, L., Conti, G., et al. 1997b, A\&A, 122, 327

Brown, E. F. 2000, ApJ, 531, 988

Brown, E. F. 2004, ApJ, 614, L57

Brown, E. F., Bildsten, L., \& Chang, P. 2002, ApJ, 574, 920

Chelovekov, I. V., Grebenev, S. A., \& Sunyaev, R. A. 2007, ArXiv e-prints, 709

Chou, Y., \& Grindlay, J. E. 2001, ApJ, 563, 934

Cooper, R. L., \& Narayan, R. 2005, ApJ, 629, 422

Cooper, R. L., \& Narayan, R. 2007, ApJ, 657, L29

Cornelisse, R., Heise, J., Kuulkers, E., Verbunt, F., \& in 't Zand, J. J. M. 2000, A\&A, 357, L21

Cornelisse, R., in 't Zand, J. J. M., Verbunt, F., et al. 2003, A\&A, 405, 1033

Cumming, A., \& Bildsten, L. 2001, ApJ, 559, L127

Cumming, A., \& Macbeth, J. 2004, ApJ, 603, L37

Cumming, A., Macbeth, J., in 't Zand, J. J. M., \& Page, D. 2006, ApJ, 646, 429 den Hartog, P. R., in 't Zand, J. J. M., Kuulkers, E., et al. 2003, A\&A, 400, 633 Fender, R., Belloni, T., \& Gallo, E. 2005, Ap\&SS, 300, 1

Fiore, F., Guainazzi, M., \& Grandi, P. 1999, Cookbook for BeppoSAX NFI Spectral Analysis, Version 1.2

Fraser, G. W. 1989, X-ray detectors in astronomy (Cambridge and New York: Cambridge University Press), 312

Frontera, F., Costa, E., dal Fiume, D., et al. 1997, A\&A, 122, 357

Galloway, D. K., Muno, M. P., Hartman, J. M., et al. 2006,

[arXiv:astro-ph/0608259]

Gierliński, M., \& Done, C. 2002, MNRAS, 337, 1373

Grindlay, J. E., \& Liller, W. 1978, ApJ, 220, L127

Gupta, S., Brown, E. F., Schatz, H., Möller, P., \& Kratz, K.-L. 2007, ApJ, 662, 1188

Haensel, P., \& Zdunik, J. L. 1990, A\&A, 227, 431

Hansen, J.-P., McDonald, I. R., \& Pollock, E. L. 1975, Phys. Rev. A, 11, 1025

Heger, A., Cumming, A., \& Woosley, S. E. 2007, ApJ, in press

Hua, X.-M., \& Titarchuk, L. 1995, ApJ, 449, 188

in 't Zand, J. J. M., Kuulkers, E., Verbunt, F., Heise, J., \& Cornelisse, R. 2003, A\&A, 411, L487

in 't Zand, J. J. M., Cornelisse, R., \& Cumming, A. 2004, A\&A, 426, 257

in 't Zand, J. J. M., Jonker, P. G., \& Markwardt, C. B. 2007, A\&A, 465, 953

Jager, R., Mels, W. A., Brinkman, A. C., et al. 1997, A\&A, 125, 557

Jahoda, K., Markwardt, C. B., Radeva, Y., et al. 2006, ApJS, 163, 401

Kaaret, P., Prieskorn, Z., Zand, J. J. M. I., et al. 2007, ApJ, 657, L97

Keek, L., in 't Zand, J. J. M., \& Cumming, A. 2006, A\&A, 455, 1031

Kirsch, M. G., Briel, U. G., Burrows, D., et al. 2005, in UV, X-Ray, and Gamma-

Ray Space Instrumentation for Astronomy XIV, ed. O. H. W. Siegmund, Proc. Spie, 589822

Kuulkers, E. 2002, A\&A, 383, L5

Kuulkers, E. 2004, Nucl. Phys. Proc. Suppl., 132, 466

Kuulkers, E. 2005, The Astronomer's Telegram, 483, 1

Kuulkers, E., Homan, J., van der Klis, M., Lewin, W. H. G., \& Méndez, M. 2002a, A\&A, 382, 947

Kuulkers, E., in 't Zand, J. J. M., van Kerkwijk, M. H., et al. 2002b, A\&A, 382, 503

Lebrun, F., Leray, J. P., Lavocat, P., et al. 2003, A\&A, 411, L141

Leinson, L. B., \& Pérez, A. 2006, Phys. Lett. B, 638, 114

Levine, A. M., Bradt, H., Cui, W., et al. 1996, ApJ, 469, L33

Lewin, W. H. G., van Paradijs, J., \& Taam, R. E. 1993, Space Sci. Rev., 62, 223

Lochner, J. C., \& Roussel-Dupre, D. 1994, ApJ, 435, 840

Makishima, K., Maejima, Y., Mitsuda, K., et al. 1986, ApJ, 308, 635

Maraschi, L., \& Cavaliere, A. 1977, in Highlights in Astronomy, ed. E. A. Müller (Dordrecht: Reidel), 4, 127

Mitsuda, K., Inoue, H., Koyama, K., et al. 1984, PASJ, 36, 741

Morrison, R., \& McCammon, D. 1983, ApJ, 270, 119

Murakami, T., Inoue, H., Koyama, K., et al. 1980, ApJ, 240, L143

Parmar, A. N., Martin, D. D. E., Bavdaz, M., et al. 1997, A\&A, 122, 309

Remillard, R., Morgan, E., \& The ASM Team at MIT, N. 2005, The Astronomer's Telegram, 482, 1

Revnivtsev, M., Churazov, E., Gilfanov, M., \& Sunyaev, R. 2001, A\&A, 372, 138

Ricker, G. R., Atteia, J.-L., Crew, G. B., et al. 2003, in American Institute of Physics Conf. Ser., Gamma-Ray Burst and Afterglow Astronomy 2001: A Workshop Celebrating the First Year of the HETE Mission, ed. G. R. Ricker \& R. K. Vanderspek, 662, 3

Rutledge, R. E., Bildsten, L., Brown, E. F., Pavlov, G. G., \& Zavlin, V. E. 1999, ApJ, 514, 945

Rutledge, R. E., Bildsten, L., Brown, E. F., Pavlov, G. G., \& Zavlin, V. E. 2000, ApJ, 529, 985

Schatz, H., Bildsten, L., Cumming, A., \& Ouelette, M. 2003, Nucl. Phys. A, 718, 247

Shirasaki, Y., Kawai, N., Yoshida, A., et al. 2003, PASJ, 55, 1033

Sidoli, L., Parmar, A. N., Oosterbroek, T., et al. 2001, A\&A, 368, 451 
Spitkovsky, A., Levin, Y., \& Ushomirsky, G. 2002, ApJ, 566, 1018

Strohmayer, T., \& Bildsten, L. 2006, New views of thermonuclear bursts (Cambridge, UK: Cambridge University Press), 113

Strohmayer, T. E., \& Brown, E. F. 2002, ApJ, 566, 1045

Strohmayer, T. E., \& Markwardt, C. B. 2002, ApJ, 577, 337

Swank, J. H., Becker, R. H., Boldt, E. A., et al. 1977, ApJ, 212, L73

Tananbaum, H., Chaisson, L. J., Forman, W., Jones, C., \& Matilsky, T. A. 1976, ApJ, 209, L125

Titarchuk, L. 1994, ApJ, 434, 570

Titarchuk, L., \& Lyubarskij, Y. 1995, ApJ, 450, 876

Ubertini, P., Lebrun, F., Di Cocco, G., et al. 2003, A\&A, 411, L131 van Paradijs, J., Penninx, W., \& Lewin, W. H. G. 1988, MNRAS, 233, 437

van Straaten, S., van der Klis, M., \& Méndez, M. 2003, ApJ, 596, 1155

Verner, D. A., Ferland, G. J., Korista, K. T., \& Yakovlev, D. G. 1996, ApJ, 465, 487

Wachter, S., Hoard, D. W., Bailyn, C. D., Corbel, S., \& Kaaret, P. 2002, ApJ, 568,901

Wilms, J., Allen, A., \& McCray, R. 2000, ApJ, 542, 914

Winkler, C., Courvoisier, T. J.-L., Di Cocco, G., et al. 2003, A\&A, 411, L1

Woosley, S. E., \& Taam, R. E. 1976, Nature, 263, 101

Woosley, S. E., Heger, A., Cumming, A., et al. 2004, ApJS, 151, 75

Yakovlev, D. G., Kaminker, A. D., \& Levenfish, K. P. 1999, A\&A, 343, 650 